\title{
GENERALIZATIONS OF PALEY-WIENER'S THEOREM FOR ENTIRE FUNCTIONS OF EXPONENTIAL TYPE
}

\author{
SABUROU SAITOH
}

\begin{abstract}
An interpretation and generalizations of the Paley-Wiener theorem for entire functions of exponential type are given in connection with the Fourier-Laplace transform.
\end{abstract}

1. Introduction. The important generalization of the Paley-Wiener theorem [6] for entire functions of exponential type was given by Plancherel and Pólya [7] (see Fuks [3] and Ronkin [8]). A further extension of Plancherel and Pólya was given by Martin [5] in the case of functions $f$ analytic on the octant $\operatorname{Im} z_{k}>0$ $(k=1,2, \ldots, n)$. In this paper, we discuss Martin's theorem in a general situation following the idea of the general theory $[9,10,11]$ of integral transforms.

The author wishes to thank Professor T. G. Genchev for pleasant and fruitful discussions in Bulgaria.

2. Fourier-Laplace transform and a fundamental problem. We set

$$
\begin{gathered}
z=\left(z_{1}, z_{2}, \ldots, z_{n}\right) \in \mathbf{C}^{n}, \\
z_{j}=x_{j}+i y_{j} \quad\left(x_{j}, y_{j} \in R\right), \\
x=\left(x_{1}, x_{2}, \ldots, x_{n}\right), \quad y=\left(y_{1}, y_{2}, \ldots, y_{n}\right) \in R^{n}, \\
d x=d x_{1} d x_{2} \cdots d x_{n}, \quad d y=d y_{1} d y_{2} \cdots d y_{n}, \\
t=\left(t_{1}, t_{2}, \ldots, t_{n}\right) \in R^{n}, \quad(z, t)=\sum_{j=1}^{n} z_{j} t_{j} .
\end{gathered}
$$

For some domains $D \subset R^{n}, \Omega \subset \mathbf{C}^{n}$, and for functions $F$ of $L_{2}(D, d t)$, we consider the Fourier-Laplace transform

$$
f(z)=\left(\frac{1}{2 \pi}\right)^{n / 2} \int_{D} F(t) e^{-i(z, t)} d t .
$$

When we consider the images $f(z)$ of $L_{2}(D, d t)$ functions $F$ by $(2.1)$, we should consider the following expression:

$$
K(z, \bar{w} ; \Omega, D)=\left(\frac{1}{2 \pi}\right)^{n} \int_{D} e^{-i(z, t)} e^{i(\bar{w}, t)} d t .
$$

Received by the editors December 18, 1985.

1980 Mathematics Subject Classification (1985 Revision). Primary 32A07, 42B10, 44A10; Secondary $30 \mathrm{C} 40$.

Key words and phrases. Paley-Wiener's theorem, entire function of exponential type, FourierLaplace transform, general theory of integral transforms, reproducing kernel, tube domain, convex domain.

Research partially supported by Grant-in-Aid for Scientific Research 60540086. 
See the general theory $[\mathbf{9}, \mathbf{1 0}, \mathbf{1 1}]$ of integral transforms for this idea. Thus, we first assume that

$$
\int_{D} e^{2(y, t)} d t<\infty \text { on } \Omega
$$

Note that (2.2) exists on $\Omega \times \bar{\Omega}$ if and only if (2.3) is valid and, further, the existence domain of $K(z, \bar{w} ; \Omega, D)$ is independent of $x$. Hence, we can naturally consider $\Omega$ as a tube domain of the form $T_{G}=R^{n}+i G \subset \mathbf{C}^{n}$. Moreover, we set

$$
\hat{G}_{D}=\left\{y \in R^{n}, \int_{D} e^{2(y, t)} d t<\infty\right\}
$$

and we can consider the maximal domain $T_{\hat{G}_{D}}$ as $\Omega$. Of course, $\hat{G}_{D}$ is a convex domain on $R^{n}$. We thus consider the function $K\left(z, \bar{w} ; T_{\hat{G}_{D}}, D\right)$ on $T_{\hat{G}_{D}} \times \bar{T}_{\hat{G}_{D}}$. This function is a positive matrix on $T_{\hat{G}_{D}}$ in the sense of E. M. Moore and so there exists a uniquely determined Hilbert space $H_{K}\left(T_{\hat{G}_{D}}, D\right)$ composed of functions on $T_{\hat{G}_{D}}$ admitting the reproducing kernel $K\left(z, \bar{w} ; T_{\hat{G}_{D}}, D\right)$ (see Aronszajn [1, 2]). This space $H_{K}\left(T_{\hat{G}_{D}}, D\right)$ is composed of holomorphic functions $f(z)$ on $T_{\hat{G}_{D}}$ which are expressible in the form

$$
f(z)=\left(\frac{1}{2 \pi}\right)^{n / 2} \int_{D} F(t) e^{-i(z, t)} d t
$$

for $L_{2}(D, d t)$ functions $F$ and with the norm

$$
\|f\|_{H_{K}\left(T_{\hat{G}_{D}}, D\right)}^{2}=\int_{D}|F(t)|^{2} d t
$$

$[9,10,11]$. On the other hand, from Parseval's equation we have

$$
\int_{R^{n}}|f(x)|^{2} d x=\int_{D}|F(t)|^{2} d t .
$$

Hence,

$$
\|f\|_{H_{K}\left(T_{\hat{G}_{D}}, D\right)}^{2}=\int_{R^{n}}|f(x)|^{2} d x .
$$

We thus see that the functions $f(z)$ of $H_{K}\left(T_{\hat{G}_{D}}, D\right)$ are analytic on $T_{\hat{G}_{D}}, L_{2}\left(R^{n}, d x\right)$ integrable, and the norms of $f$ in $H_{K}\left(T_{\hat{G}_{D}}, D\right)$ are given by (2.8). In this situation, we can regard that when $D$ is a bounded interval in $R$ and when $D$ is a bounded convex domain in $R^{n}$, the theorems of Paley-Wiener [6] and Plancherel-Pólya [7] give characterizations of the members $f(z)$ of $H_{K}\left(T_{\hat{G}_{D}}, D\right)$ in terms of the growth of $f(z)$ at infinity, respectively. We thus, in general, propose a fundamental problem in our situation.

$A$ fundamental problem. In the above situation, give a characterization of the members of $H_{K}\left(T_{\hat{G}_{D}}, D\right)$ in terms of the domain $D$.

In order to give a reasonable solution for this problem, we will assume that $D$ is a convex domain and $\partial D$ is a smooth hypersurface on $R^{n}$. When $D=\prod_{j=1}^{n}(-\infty, a)$ $(a>0)$, Martin [5] discussed the growth of the functions of $H_{K}\left(T_{\hat{G}_{D}}, D\right)$ at infinity, but he did not give a complete answer for the above problem in his situation. 
3. On ranges. Let $O$ be the origin of coordinates in the $t$-space $R^{n}$ and let the hyperplanes $\{\Gamma\}$ pass through it and lie parallel to the limiting positions of the tangent hyperplanes of $\partial D$. We consider the convex cone with vertex at the origin enveloped by these hyperplanes. The nappe of this cone lying on the same side of the hyperplanes $\{\Gamma\}$ as the domain $D$ is called the asymptotic cone of $T_{D}$. As the asymptotic cone of a bounded domain $D$, we take the set $\{0\}$; that is, the origin. When $V$ is the asymptotic cone of $T_{D}$, we will say that the domain $T_{D}$ is of type $V$.

We consider the conjugate cone $V^{*}$ of $V$; that is,

$$
V^{*}=\left\{\left(t_{1}^{*}, t_{2}^{*}, \ldots, t_{n}^{*}\right) \in R^{n} ; \sum_{j=1}^{n} t_{j}^{*} t_{j}>0 \text { for all } t \in \bar{V}, t \neq 0\right\} .
$$

When $D$ contains a whole line, $V^{*}$ does not contain any $n$-dimensional sphere. Further then, since $\hat{G}_{D}=\{\emptyset\}$, in the sequel we assume that $D$ does not contain any whole line.

We will consider $V^{*}$ in the $y$-space $R^{n}$. Then, note that

$$
e^{(y, t)} \text { is bounded on } t \in D
$$

if and only if

$$
-y \in V^{*} \text { or } y \in-V^{*} \text {. }
$$

We define the support function of the convex set $\bar{D}$ by

$$
H_{D}(y)=\max _{t \in \bar{D}}(y, t)
$$

Then we obtain

THEOREM 3.1. $\hat{G}_{D}=-V^{*}$.

Proof. For any fixed point $y^{(0)} \in-V^{*}$, we set

$$
\left(y^{(0)}, t\right)=\left|y^{(0)}\right||t| \cos \theta_{0}(t)<0 \quad \text { on } V,
$$

where, of course, $\theta_{0}(t)\left(\left|\theta_{0}(t)\right| \leq \pi\right)$ is the angle between the two vectors $y^{(0)}$ and $t$ in the same space $R^{n}$. Hence, there exists $\Theta$ such that

$$
\left|\theta_{0}(t)\right| \geq \Theta>\pi / 2 \text { on } V .
$$

Hence, there exist $\varepsilon>0$ and $M>0$ such that

$$
\left|\theta_{0}(t)\right| \geq \Theta-\varepsilon>\pi / 2 \text { on } D \cap\{|t| \geq M\} .
$$

Then, from the identity

$$
\int_{|x|<N} f(|x|) d x=\frac{2 \sqrt{\pi^{n}}}{\Gamma(n / 2)} \int_{0}^{N} x^{n-1} f(x) d x
$$


[4, p. 623], we have

$$
\begin{aligned}
\int_{D} e^{2\left(y^{(0)}, t\right)} d t \leq & \int_{D \cap\{|t| \leq M\}} e^{2\left(y^{(0)}, t\right)} d t+\int_{D \cap\{|t| \geq M\}} e^{2\left|y^{(0)}\right||t| \cos (\Theta-\varepsilon)} d t \\
\leq & \int_{D \cap\{|t| \leq M\}} e^{2\left(y^{(0)}, t\right)} d t+\int_{R^{n}} e^{2\left|y^{(0)}\right||t| \cos (\Theta-\varepsilon)} d t \\
\leq & \int_{D \cap\{|t| \leq M\}} e^{2\left(y^{(0)}, t\right)} d t \\
& +\lim _{N \rightarrow \infty} \frac{2 \sqrt{\pi^{n}}}{\Gamma(n / 2)} \int_{0}^{N} \cdot t^{n-1} e^{t\left[2\left|y^{(0)}\right| \cos (\Theta-\varepsilon)\right]} d t \\
< & \infty
\end{aligned}
$$

Hence, we have $\hat{G}_{D} \supset-V^{*}$.

On the other hand, for any point $y^{(0)} \in\left(-V^{*}\right)^{c}$, the complement, by the definition of $V^{*}$ there exists a point $t^{(0)} \in V$ such that $\left(y^{(0)}, t^{(0)}\right)>0$. Then, there exists a narrow nondegenerate (i.e. contains an $n$-dimensional sphere) convex cone $\Gamma\left(t^{(0)}\right)$ with vertex 0 such that

$$
\left(y^{(0)}, t\right)>0 \text { on } \Gamma\left(t^{(0)}\right)
$$

and

$$
D \supset \Gamma\left(t^{(0)}\right) \cap\{|t| \geq M\} \text { for some } M>0 .
$$

Then

$$
\begin{aligned}
\int_{D} e^{2\left(y^{(0)}, t\right)} d t & \geq \int_{\Gamma\left(t^{(0)}\right) \cap\{|t| \geq M\}} e^{2\left(y^{(0)}, t\right)} d t \\
& \geq \int_{\Gamma\left(t^{(0)}\right) \cap\{|t| \geq M\}} d t=\infty .
\end{aligned}
$$

Hence, $\hat{G}_{D} \subset-V^{*}$, and we have the desired result.

In Theorem 3.1, we will give a characterization of the functions $f \in$ $H_{K}\left(T_{-V^{*}}, D\right)$.

\section{Necessity condition. We set}

$$
y=\rho \lambda \quad\left(y_{j}=\rho \lambda_{j}, \rho>0\right) \quad \text { and } \quad|\lambda|=1 .
$$

From the identity (2.5), we have, by Parseval's equation,

$$
\begin{aligned}
\int_{R^{n}}|f(x+i \rho \lambda)|^{2} d x & =\int_{D}|F(t)|^{2} e^{2 \rho(\lambda, t)} d t \\
& \leq e^{2 H_{D}(\lambda)} \int_{D}|F(t)|^{2} d t .
\end{aligned}
$$

Hence, for $\rho \lambda \in-V^{*}$ we have

$$
\frac{1}{2} \varlimsup_{\rho \rightarrow \infty} \frac{1}{\rho} \log \int_{R^{n}}|f(x+i \rho \lambda)|^{2} d x \leq H_{D}(\lambda) .
$$

Further, when $f(t) \neq 0$ a.e. on $D$ we can prove that the actual limit exists and that it is equal to $H_{D}(\lambda)$ as in Martin [5]. 
5. Sufficiency condition. In our situation, we will give a complete answer for our fundamental problem:

THEOREM 5.1. $f(z)$ belongs to $H_{K}\left(T_{-V^{*}}, D\right)$ if and only if

(5.1) $f(z)$ is holomorphic on $T_{-V^{*}}$ and $L_{2}\left(R^{n}, d x\right)$-integrable, and fur-

ther, for any $y \in-V^{*}$, the integral

$$
\int_{R^{n}}|f(x+i y)|^{2} d x
$$

exists,

and

$$
\frac{1}{2} \varlimsup_{\substack{\rho \rightarrow \infty \\ \rho \lambda \in-V^{*}}} \frac{1}{\rho} \log \int_{R^{n}}|f(x+i \rho \lambda)|^{2} d x \leq H_{D}(\lambda) .
$$

PROOF. It is sufficient to prove that any function $f(z)$ satisfying (5.1) and (5.2) is an image by the Fourier-Laplace transform (2.1) of an $L_{2}(D, d t)$ function. Since $f(x) \in L_{2}\left(R^{n}, d x\right)$, we can define the $L_{2}\left(R^{n}, d t\right)$ function $\tilde{F}(t)$ by

$$
\tilde{F}(t)=\underset{N \rightarrow \infty}{\lim }\left(\frac{1}{2 \pi}\right)^{n / 2} \int_{\left|x_{j}\right|<N} f(x) e^{i(x, t)} d x .
$$

Of course,

$$
f(x)=\underset{N \rightarrow \infty}{\operatorname{iim}}\left(\frac{1}{2 \pi}\right)^{n / 2} \int_{\left|t_{j}\right|<N} \tilde{F}(t) e^{-i(x, t)} d t,
$$

in the framework of $L_{2}$ spaces.

We first assume that in addition $f(x) \in L_{1}\left(R^{n}, d x\right)$. Then, (5.3) exists in the ordinary sense

$$
\tilde{F}(t)=\left(\frac{1}{2 \pi}\right)^{n / 2} \int_{R^{n}} f(x) e^{i(x, t)} d x .
$$

By condition (5.2), since the integrals $\int_{R^{n}}|f(x+i y)|^{2} d x$ exist for all $y \in-V^{*}$, the integrals

$$
\left(\frac{1}{2 \pi}\right)^{n / 2} \int_{R^{n}} f(x+i y) e^{i(x+i y, t)} d x
$$

also exist for all $y \in-V^{*}$. Moreover, by using the Cauchy integral theorem, we see that the integrals (5.5) are independent of $y \in-V^{*}$. Hence, we set

$$
\tilde{\tilde{F}}(t)=\left(\frac{1}{2 \pi}\right)^{n / 2} \int_{R^{n}} f(x+i y) e^{i(x+i y, t)} d x .
$$

(See [12, pp. 98-101] for this argument.) Then, we see immediately that $\tilde{\tilde{F}}(t)$ is continuous on $R^{n}$ and $\tilde{\tilde{F}}(t)=\tilde{F}(t)$ on $R^{n}$. Hence, by Parseval's equation, we have

$$
\int_{R^{n}}|f(x+i y)|^{2} d x=\int_{R^{n}}|\tilde{F}(t)|^{2} e^{2(y, t)} d t .
$$


Hence, for $y=\rho \lambda \in-V^{*}$, we have

$$
\begin{aligned}
H_{D}(\lambda) & \geq \frac{1}{2} \varlimsup_{\rho \rightarrow \infty} \frac{1}{\rho} \log \int_{R^{n}}|f(x+i \rho \lambda)|^{2} d x \\
& =\frac{1}{2} \varlimsup_{\rho \rightarrow \infty} \frac{1}{\rho} \log \int_{R^{n}}|\tilde{F}(t)|^{2} e^{2 \rho(\lambda, t)} d t .
\end{aligned}
$$

For any $t_{0} \in \bar{D}^{c}$, we will show that $\tilde{F}\left(t_{0}\right)=0$. Since $\tilde{F}(t)$ is continuous on $R^{n}$, when $\tilde{F}\left(t_{0}\right) \neq 0$, for some closed sphere $\overline{S\left(t_{0}\right)}$ with the center $t_{0}\left(\subset \bar{D}^{c}\right)$,

$$
|\tilde{F}(t)| \geq m>0 \text { on } \overline{S\left(t_{0}\right)} \text { for some constant } m \text {. }
$$

Further then, since

$$
(\lambda, t)>H_{D}(\lambda) \text { on } \overline{S\left(t_{0}\right)}
$$

there exists $\varepsilon>0$ such that

$$
(\lambda, t) \geq H_{D}(\lambda)+\varepsilon \quad \text { on } \overline{S\left(t_{0}\right)}
$$

Hence, from (5.6) we have

$$
\begin{aligned}
H_{D}(\lambda) & \geq \frac{1}{2} \lim _{\rho \rightarrow \infty} \frac{1}{\rho} \log \int_{\overline{S\left(t_{0}\right)}}|\tilde{F}(t)|^{2} e^{2 \rho(\lambda, t)} d t \\
& \geq \frac{1}{2} \lim _{\rho \rightarrow \infty} \frac{1}{\rho} \log \left\{m^{2} e^{2 \rho\left(H_{D}(\lambda)+\varepsilon\right)} \int_{\overline{S\left(t_{0}\right)}} d t\right\} \\
& =H_{D}(\lambda)+\varepsilon
\end{aligned}
$$

which implies a contradiction. Hence, the support of $\tilde{F}$ is contained in $\bar{D}$. Further, since (2.3) is valid for any $y \in-V^{*}$, in (5.4) we obtain the desired expression

$$
f(z)=\left(\frac{1}{2 \pi}\right)^{n / 2} \int_{D} \tilde{F}(t) e^{-i(z, t)} d t .
$$

When $f(x)$ is in $L_{2}\left(R^{n}, d x\right)$ but not necessarily in $L_{1}\left(R^{n}, d x\right)$ we set

$$
f_{\varepsilon}(z)=f(z) \prod_{j=1}^{n} \frac{\sin \varepsilon z_{j}}{\varepsilon z_{j}} .
$$

Then we have $f_{\varepsilon} \in L_{1}\left(R^{n}, d x\right)$. Hence, the Fourier transform $\tilde{F}_{\varepsilon}(t)$ of $f_{\varepsilon}(z)$ vanishes outside the convex set $\bar{D}$. From the relation

we obtain

$$
\tilde{F}_{\varepsilon}(t)=\left(\frac{1}{2 \varepsilon}\right)^{n} \int_{t_{1}-\varepsilon}^{t_{1}+\varepsilon} \cdots \int_{t_{n}-\varepsilon}^{t_{n}+\varepsilon} \tilde{F}(\hat{t}) d \hat{t}
$$

$$
\lim _{\varepsilon \rightarrow 0} \tilde{F}_{\varepsilon}(t)=\tilde{F}(t)
$$

for almost all $t \in R^{n}$. Hence, the support of $\tilde{F}$ is contained in $\bar{D}$. We thus complete the proof of the theorem.

ADDED IN PROOF. In Theorem 5.1, $f(x)$ are also considered as the boundary values such that

$$
\lim _{\substack{y \rightarrow 0 \\ y \in-V^{*}}} f(x+i y)=f(x)
$$

in the sense of the $L_{2}$ norm (see [12, Chapter III]). 


\section{REFERENCES}

1. N. Aronszajn, La theorie des noyaux reproduisants et ses applications. I, Proc. Cambridge Philos. Soc. 39 (1943), 113-153.

2. __ Theory of reproducing kernels, Trans. Amer. Math. Soc. 68 (1950), 337-404.

3. B. A. Fuks, Introduction to the theory of analytic functions of several complex variables, Fizmatgiz, Moscow, 1962; English transl., Transl. Math. Mono., Vol. 8, Amer. Math. Soc., Providence, R.I., 1963.

4. I. S. Gradshteyn and I. M. Ryzhik, Tables of integrals, series, and products, Academic Press, New York, 1980.

5. W. T. Martin, Analytic functions and multiple Fourier integrals, Amer. J. Math. 62 (1940), 673679.

6. R. E. A. C. Paley and N. Wiener, Fourier transforms in the complex plane, Amer. Math. Soc. Colloq. Publ., Vol. 19, Amer. Math. Soc., Providence, R.I., 1934.

7. M. Plancherel and G. Pólya, Founctions entières et integrals de Fourier multiple, Comment. Math. Helv. 9 (1936-37), 224-248; 10 (1937-38), 110-163.

8. L. I. Ronkin, Introduction to the theory of entire functions of several variables, Transl. Math. Mono., Vol. 44, Amer. Math. Soc., Providence, R.I., 1974.

9. S. Saitoh, Integral transforms in Hilbert spaces, Proc. Japan Acad. Ser. A Math. Sci. 58 (1982), 361-364.

10. __ Hilbert spaces induced by Hilbert space valued functions, Proc. Amer. Math. Soc. 89 (1983), 74-78.

11. _ A general theory of integral transforms and its applications, Math. Vesnik 37 (1985), 121-133.

12. E. M. Stein and G. Weiss, Introduction to Fourier analysis on Euclidean spaces, Princeton Univ. Press, Princeton, N.J., 1975.

Department of Mathematics, FaCulty of Engineering, Gunma University, KIRYU, 376, JAPAN

Current address: Department of Mathematics and Statistics, University of Pittsburgh, Pittsburgh, Pennsylvania 15260 